\title{
La Valoración de Empresas en la Economía Social. Valor Social Esperado del Museo de la Minería del País Vasco
}

\section{Iratxe Amiano, Leire San-Jose, Jorge Gutiérrez Goiria}

RESUMEN: Las técnicas de valoración de empresas se centran en aspectos económico-financieros, que no resultan suficientes para las entidades no lucrativas, cuyas prioridades se asocian a la consecución de un interés general. Hasta ahora, diversas técnicas de contabilidad social como el Valor Social Integrado (VSI) han servido para reflejar estas inquietudes en las entidades no lucrativas. En este artículo, utilizando un museo como caso ilustrativo, se va a demostrar cómo es posible la valoración de las entidades no lucrativas utilizando la contabilidad social, y aprovechando al tiempo (de forma adaptada) las bases de las técnicas de valoración de empresas. Los principales resultados demuestran, en primer lugar, que el Valor Social Integrado es una variable monetizable, y por tanto útil y sencilla para realizar valoraciones de entidades. Aunque esta variable puede ser utilizada para todo tipo de organizaciones, para las no lucrativas es esencial ya que permite la comparación de su dimensión social, más acorde con su propósito como entidades. La principal contribución consiste en demostrar que es posible calcular el Valor Social Esperado (VSE, mediante la proyección y actualización de VSI) para valorar entidades de todo tipo. Esto tiene también implicaciones en las teorías relacionadas con la valoración de empresas, que podrán tener en cuenta variables más allá de las meramente económicas o transaccionales, y adaptarse a las situaciones actuales donde lo social es una variable más a valorar por parte de los decisores, tanto estratégicos, institucionales, como inversionistas.

PALABRAS CLAVE: Monetización del valor social, Valoración de Empresas, Valor Social Esperado (VSE), Entidades no Lucrativas, Indicadores de la Economía Social y Contabilidad Social.

CLAVES ECONLIT: M40, M14, 035, A13.

Cómo citar este artículo/How to cite this article: AMIANO, I., SAN-JOSE, L. \& GUTIÉRREZ, J. (2021): "La Valoración de Empresas en la Economía Social. Valor Social Esperado del Museo de la Minería del País Vasco", CIRIEC-España, Revista de Economía Pública, Social y Cooperativa, 101, 33-56. D0I: 10.7203/ CIRIEC-E.101.18226.

Correspondencia: Iratxe Amiano, Universidad del País Vasco (UPV/EHU), iratxe.amiano@ehu.eus, ORCID: 0000-0003-4139-4168; Leire San-Jose, Universidad del País Vasco (UPV/EHU), University of Huddersfield (UK), leire.sanjose@ehu.eus, ORCID: 0000-0003-2760-3285; Jorge Gutiérrez Goiria, Universidad del País Vasco (UPV/EHU), jorge.gutierrez@ehu.eus, ORCID: 0000-0002-5558-3253. 


\section{Expanded abstract}

\section{Company Valuation in entities of the Social Economy. Expected Social Value of the Basque Country Mining Museum}

The generation of social value is an aspect that has gained importance, not only in social entities but also in commercial ones. Despite this, some instruments, such as the valuation of companies, do not consider the inclusion of a social perspective. This perspective is important for all entities, but especially for non-profit organizations, since their purpose is primarily social and not that of generating economic value.

The aim of this paper is to respond to this lack of social inclusion in the area of business valuation. This paper demonstrates how a social value can be introduced and measured through the Integrated Social Value (ISV) of social accounting, as a differential factor in business valuation models. Thus, the Expected Social Value (ESV) is calculated from the ISV, which is incorporated into the calculation for updating future flows. Incorporating variables beyond the classic economic-financial ones in the valuation of companies allows any entity to show its social valuation in a more comprehensive way, but especially those in the social economy whose primary function is of a social nature. Progress towards this approach will encourage decision-making based on social aspects, not just economic ones.

This proposal, which is framed in social accounting, monetizes the social value of organizations from the perspective of the stakeholders, that is, in a holistic way with the perspective of those who are the beneficiaries of the social value (see Retolaza et al., 2016; San-Jose and Retolaza, 2016 and Retolaza and San-Jose, 2018, for a more complete explanation).

The methodology used is that of a case study, specifically, that of the Basque Country Mining Museum. In 2016, the museum undertook a training process using action research methodology called: "Action research Training: (Social) Assessment of the Basque Country Mining Museum", which aimed to incorporate the process of social accounting known as the SPOLY methodology to quantify the value of the museum from a social perspective. The data obtained at that time have been updated in order to present results adapted to the current context. Thus, calculations have been made based on the values corresponding to the last three fiscal years, that is, 2017, 2018 and 2019, and these are used to calculate the Integrated Social Value of three years $(2017,2018$ and 2019). These values have then been projected for the following 5 years, a continuity value estimated, and any necessary update or discount of future flows carried out.

In this way, the valuation of the museum has been carried out from a socially generated and expected value, and not based solely on economic-financial variables. Thus, the social income measured through the ISV, which includes the market and non-market value, can be used to 
establish not only what an entity is generating socially, but what it can generate for the future. Its update gives us the Expected Social Value, that is, the value of the museum as an entity, this being a measure more in line with its social purpose.

The results obtained from the application of the methodology lead us to conclude that the museum's value stands at $€ 11,453,652 €$ based on a long-term horizon of 15 years. It should be noted that the museum has been operating for more than 15 years, and at all times we have worked within prudent guidelines with the continuity of its work based on current parameters.

We conclude that it is possible, and advantageous, to use company valuation techniques in non-profit entities; the valuation can be used in terms of communication and management, as well as for building trust in the social value, thus showing a commitment to society.

We also contribute to the valuation theories of companies by opening horizons towards social rather than just economic-financial aspects, generating value towards stakeholders (workers, suppliers, clients) reflected in the creation of value for society, and not just from the perspective of value generated for those who contribute capital. Finally, it should be noted that this proposal should not be limited to social enterprises. This paper opens the way to using this adaptation of the valuation of companies not only in non-profit entities but also in all types of entities, regardless of their legal form, their social character, their governance or their public affiliation.

The contribution of this paper has been twofold. On the one hand, our contribution has academic value since it demonstrates that it is possible to carry out assessments using not only economic but also social variables. On the other hand, it offers a practical contribution, since it enables the expected social value of an entity to be determined through social accounting, leading to a clearer understanding of the role of the entity, and, therefore, enabling comparisons to be made with other companies in a dimension other than an economic one.

The main limitation to this study comes from the fact that it is based on only one case study. In addition, the ISV itself has limitations, one of which is the use of proxies to monetize the variables of social value generated for stakeholders. Also, it may be necessary to analyse whether there are other social income variables that can be applied in a complementary way, or might substitute those proposed. In addition, issues such as the discount rate to be used in the assessment of non-profit organizations can be open to debate and, therefore, may require a more in-depth analysis.

Regarding future lines of research, firstly, mechanisms should be established to agree on the proxies to be used. For this, work groups or user communities could be effective, with a sample of entities from each sector participating, in order to reach consensus on the amounts to use and apply. Secondly, it would be desirable to have more organizations who apply these valuation techniques in order to confirm that the Expected Social Value is applicable in different organizations. Finally, a future analysis is recommended of the impact on non-profit organizations that a change of perspective such as this could have as a result of other entities being valued in terms of their social value. This could help to influence global understanding, and offer other approaches in terms of valued generated between mercantile companies and the social 
economy, which to date has simply been seen as economic. The Expected Social Value may thus be a mechanism for communication, future projection or global comparison.

Keywords: Monetization of Social Value, Valuation of firms, Non-Profit Organizations, Expected Social Value (ESV), Social Economy Indicators and Social Accounting. 


\section{Introducción'}

La generación de valor social en las entidades es un aspecto que está adquiriendo relevancia, no solo en las entidades sociales, sino también en las mercantiles. A pesar de este incremento en su relevancia, algunos instrumentos, como la valoración de empresas, no contemplan la inclusión de la perspectiva social. Esta perspectiva es importante para todas las entidades, pero en especial para las entidades no lucrativas (ENLs) ya que su razón de ser precisamente es la social y no la de generación de valor económico. Precisamente, somos conscientes de la relevancia que esta valoración de empresas en ENLs puede llegar a tener, ya que permitirá un diálogo diferente con las administraciones públicas, podrá integrar de forma holística los valores generados y no solo los económicos y dará una visión, que probablemente conllevará una reputación social positiva, en cuanto a cuál es su propósito esperado y su responsabilidad con la sociedad. Este mecanismo de medición, además de como instrumento de comunicación, podrá ser utilizado como mediador entre diferentes decisiones. Así, siendo la perspectiva social esencial en las ENLs, no hay estudios que aborden cómo es posible aplicar la valoración de empresas a ENLs de forma completa, es decir, desde la recogida de información social para establecer el valor social hasta la forma de realizar la valoración social de la organización, mediante lo que denominaremos Valor Social Esperado (VSE).

La valoración social a partir de una contabilidad social se ha realizado ampliamente en la última década, por ello existen estudios que exponen los principios y sus evidencias (Retolaza et al., 2016), su aplicabilidad en diferentes sectores, como lo es el de las cofradías (Guzmán et al., 2020), el de cooperativas (Etxezarreta et al., 2018), en general el de economía social (Lazkano y Beraza, 2019; Lazkano y San-Jose, 2020) o el de universidades (Ayuso et al., 2020), pero no existe trabajo previo sobre su aplicación a la valoración de empresas. La incorporación de variables más allá de lo económico-financiero a las técnicas de valoración tiene gran interés y actualidad, ya que permitirá a cualquier entidad mostrar su valoración social de una forma más completa. Además, el avance en esta línea permitirá la toma de decisiones basada en otros aspectos y no solamente los económicos. Por último, los planes estratégicos y las decisiones a largo plazo pueden estar condicionadas por los datos de más de un momento concreto, es decir, de un periodo, con lo que todo avance en la valoración, y en especial, en la social, será de gran provecho para todo tipo de entidades. Así, el objetivo de este trabajo es analizar y demostrar cómo es posible introducir el valor social medido a través del Valor Social Integrado (VSI) de la contabilidad social, como elemento diferencial en los modelos de valoración de empresas, así generaremos el VSE.

Vamos a utilizar el caso del Museo de la Minería del País Vasco para demostrar su aplicabilidad y uso utilizando datos actualizados de 2017, 2018 y 2019; y realizando proyecciones a 5 años, estimando posteriormente un valor de continuidad. Así generaremos el Valor Social Esperado actualizando el VSI. Esto nos permitirá aportar un valor que ha sido calculado uti-

1. Los autores agradecen a los revisores y editores de CIRIEC sus aportes. Este trabajo ha sido financiado por la Universidad del País Vasco (UPV/EHU), proyectos US20/11 y GIU17/26. 
lizando técnicas habituales en la valoración empresarial, pero en este caso, aplicado a una entidad sin ánimo de lucro y con claros fines sociales.

La contribución de este trabajo será doble. Por un lado, nuestra aportación tiene un valor académico ya que se demuestra que es posible realizar valoraciones utilizando no solo variables económicas sino también sociales. Por otro lado, una contribución práctica ya que permite conocer y determinar el valor social esperado de las entidades a partir de la contabilidad social, lo que implica mayor conocimiento sobre estas entidades, y, por tanto, poder realizar comparaciones junto con las empresas en otra dimensión que no sea la económica. Con estos sistemas se podrán valorar de forma diferencial otro de los propósitos de las empresas, el social, lo que ayudará en el logro de entender la empresa no solamente desde el ámbito económico sino también desde el social, en línea con la Triple Bottom Line.

En los siguientes apartados abordamos estos aspectos; así en la sección dos se expone la principal literatura en relación con la valoración de empresas desde una perspectiva clásica, una perspectiva más allá de la basada en transacciones de mercado y la apertura hacia la contabilidad social. En la siguiente sección, la tercera, se describe la metodología utilizada, el análisis de caso con su aplicación y las metodologías utilizadas, el valor social integrado de la contabilidad social y las fórmulas para la valoración de empresas que nos permiten calcular el valor social generado. Posteriormente, se exponen los resultados obtenidos tras lograr el Valor Social Esperado como valoración de ENLs. Finalmente, en la última sección se exponen las conclusiones, limitaciones y futuras líneas.

\section{Marco Teórico}

\subsection{Metodologías de valoración de empresas: perspectiva clásica}

La valoración de empresas puede tener diferentes objetivos. Habitualmente están relacionados con la adquisición en todo o en parte de la entidad, mediante procesos de compra o absorción, Oferta Pública de Adquisición (OPA), salidas a bolsa, herencias u otros. En otras ocasiones la valoración se vincula a la voluntad de conocer el valor de la entidad y su evolución, como posible guía para una planificación estratégica orientada a la creación de valor.

Estos procesos buscan obtener el valor de la entidad, sin confundirlo con el precio que en un momento pueda tener, y que especialmente en el caso de las grandes empresas puede variar súbitamente según las informaciones o vaivenes del mercado. Se trata de analizar los fundamentos de la actividad, trascendiendo el enfoque coyuntural.

Para ello hay diferentes metodologías, que en casi todos los casos realizan su aporte y tienen interés para ofrecer una visión amplia del valor (ver, por ejemplo: Damodaran, 2016; Fernández, 2019). 
Los métodos basados en el balance, por ejemplo, nos ofrecen una visión parcial (ya que hay elementos intangibles que escapan a la contabilidad), estática, y sin expectativas futuras. Sin embargo, y pese a ser limitada, esta información da idea de un valor mínimo de la empresa, así como de la inversión realizada, lo que puede ser de interés para contrastar el valor finalmente obtenido, que deberá guardar cierta proporción con los medios empleados.

Otros métodos, llamados mixtos, tratan de añadir a la perspectiva estática del balance alguna perspectiva de futuro, de una forma sencilla (un número de veces el beneficio o la facturación, por ejemplo).

Hay igualmente métodos basados en múltiplos (del rendimiento, determinados valores contables, las ventas, los clientes, u otras variables clave en cada sector), que permiten de una forma intuitiva y rápida tener una idea del rango de valor de una entidad, a partir de otros casos considerados comparables.

En todo caso, el método más desarrollado y comúnmente aceptado es el que valora las entidades en función del descuento de sus flujos de caja futuros. Para autores como Fernández (2019) se trata de hecho del único método conceptualmente correcto.

Desde esta perspectiva, el valor de la empresa se calcula actualizando la corriente de flujos futuros que la misma va a generar, según la siguiente fórmula:

$$
\text { Valor }=\frac{\text { Flujo de Caja }_{1}}{(1+k)}+\frac{\text { Flujo de Caja }_{2}}{(1+k)^{2}}+\cdots+\frac{\text { Flujo de Caja }_{n}+\text { Valor Residual }_{n}}{(1+k)^{n}}
$$

La tasa $\mathrm{k}$, con la que se actualizan los flujos de caja futuros, refleja la preferencia por flujos de caja cercanos frente a los lejanos en el tiempo. Se penaliza así la obtención de resultados futuros, y se compensa el riesgo derivado de la inversión y el alejamiento en el tiempo.

Tras realizar los cálculos de los flujos de caja esperados para los n años proyectados, debe sumarse también al final el Valor Residual o de continuidad que tendrá la empresa en ese momento. Para ello hay diferentes aproximaciones, entre las cuales es habitual suponer una renta (que puede ser perpetua) con una tasa de crecimiento constante.

La asunción de esta metodología, sin embargo, no da lugar a resultados únicos. A la propia dificultad de estimar los flujos de caja futuros (generalmente partiendo de los pasados) se unen las diferentes perspectivas de cálculo de los mismos. Estos pueden calcularse como los flujos que quedan para el accionista, o bien como flujos de caja libres, más operativos, que no tienen en cuenta el endeudamiento (serían los flujos antes de intereses).

De igual modo, la determinación de la tasa de actualización condicionará en gran medida el valor resultante. Esta tasa vendrá marcada en primer lugar por el tipo de flujo utilizado: si son flujos para el accionista, se descontará a la tasa que marquen los accionistas; si son flujos de caja libres deberá tener en cuenta los costes de fondos propios y ajenos (normalmente el coste medio ponderado del capital). Actualmente no hay acuerdo ni practicas comunes en cuanto a la tasa que debe aplicarse y su método, lo que da lugar a valoraciones muy diversas.

El propio horizonte de valoración marcado supone también una decisión clave en el proceso. Un número reducido de años puede hacer que las estimaciones sean mejores en ese pe- 
ríodo, pero añade un mayor peso al valor residual, que es por definición más incierto. Debe buscarse un equilibrio en este sentido y, en caso de utilizarse una renta perpetua para estimar el valor residual, debe considerarse que la tasa de crecimiento (o no) de esa renta resultará fundamental en el valor final.

En la práctica, tal como recogen Bancel y Mittoo (2014) en una amplia muestra, cerca del $80 \%$ de analistas utilizan más de un método en sus procesos de valoración. Los métodos basados en descuento de flujos de caja son los más habituales, tras los que se encuentran los que compararan valores relativos o ratios. Sin embargo, y pese al marco común aceptado, especialmente en cuanto al descuento de flujos, las valoraciones son muy variables por las diferentes formas de calcular los parámetros implicados, y la falta de criterios comunes. Así, la dificultad para estimar la tasa de descuento y el valor residual se destacan como las mayores limitaciones de los modelos actuales de valoración en el citado estudio. Los flujos suelen estimarse para períodos de entre 5 y 10 años, en función de los planes y estrategias de la entidad, y la cuestión de la tasa de descuento está lejos de estar resuelta. En ocasiones se utilizan modelos como el Capital Asset Pricing Model (CAPM), pero es muy habitual fijar un tipo libre de riesgo más una prima, o directamente valores estimados que suelen estar entre el 8 y el 12\%, en función de los riesgos operativos o financieros.

\subsection{Metodologías de valoración de empresas: una perspectiva más allá del mercado}

Como se ha visto, la valoración de empresas tiene una trayectoria consolidada, aunque no exenta de problemas prácticos. La cuestión es algo más complicada cuando el concepto del valor se amplía más allá de una visión estrictamente económico-financiera. Así, nos enfrentamos en ocasiones a la necesidad de evaluar los impactos de las políticas públicas, valorar bienes (o males) globales, o bien estudiar el valor que aportan entidades cuyo objetivo no es generar flujos de caja, ni ser objeto de una compraventa.

Si bien estas situaciones difieren del entorno de la valoración de empresas al uso, especialmente si la finalidad de la valoración empresarial va unida a una transacción, también hay puntos comunes que pueden aprovecharse para el estudio del valor de las entidades no lucrativas. Como se ha indicado, la propia finalidad estratégica que en ocasiones tiene la valoración en las empresas puede aplicarse a la gestión de entidades no lucrativas, que con estas técnicas tratarían de mejorar su gestión, identificando las claves para mantener o aumentar su valor social.

En este marco, se añaden las dificultades propias de valorar lo que no pasa por el mercado. Para ello se utilizan por ejemplo los métodos de valoración contingentes, habituales en el caso de los bienes públicos globales (especialmente ambientales), pero también en ámbitos culturales o sociales. En estos casos las preferencias no se revelan a través de un flujo monetario, y deben inferirse realizando encuestas para ver cuánto se estaría dispuesto a pagar, ordenando preferencias etc. De este modo puede llegar a establecerse por ejemplo el valor económico de un museo, como parte del patrimonio cultural (Gómez-Zapata et al., 2018). 
Entre los aspectos diferenciales, en las organizaciones no lucrativas es clave medir el valor y contribución del voluntariado, que no se refleja en las cuentas. Para ello pueden plantearse diferentes aproximaciones, como las basadas en el coste de reemplazo (lo que costaría contratar a alguien que realice esa tarea), o las que tratan de medir la contribución del voluntariado a los ingresos de la entidad (Bowman, 2009). En la práctica se trata de una cuestión compleja, con diferentes opciones técnicas y dificultades prácticas de aplicación (Cordery et al., 2011).

Entre los métodos que se utilizan en estas ocasiones se encuentran los Análisis de Coste Beneficio, cuya perspectiva no es la de un inversor individual, sino la de una visión pública o común. Se trata de evaluar los aportes positivos y negativos de una actividad (o de varias, para compararlas), teniendo en cuenta el aspecto financiero (los flujos de caja), pero también los beneficios o cambios esperados en el bienestar desde una perspectiva social. Esta metodología, en la medida en que se proyecte en un horizonte temporal, requerirá igualmente una decisión sobre las tasas de actualización aplicables en términos financieros y sociales. En el caso de los efectos sociales o ambientales, por ejemplo, se plantea si deben descontarse los efectos a muy largo plazo, o si esto supone primar el bienestar de las generaciones presentes respecto a las futuras.

En esta línea, Tessler et al. (2019) plantean un Análisis Coste Beneficio sobre las galerías, bibliotecas, archivos y museos de Canadá. Mientras el cálculo de los costes operativos es sencillo, los beneficios resultan más complicados de medir, y se recurre a una amplia encuesta para estimar aspectos como el valor para los usuarios, y para quienes valoran la propia existencia de estas entidades (pese a no haberlos visitado recientemente), el valor educativo para estudiantes, así como otros beneficios. Su perspectiva es la de lograr un Valor Económico Total, monetizando todos esos beneficios percibidos.

Desde otra perspectiva, Wnuczak (2018) propone para las entidades culturales un cálculo del Valor Social Añadido, que sería positivo en la medida en que el Valor logrado supere al coste social del capital empleado, estableciendo así un paralelismo con el concepto del Valor Económico Agregado (EVA), que en entornos empresariales mide el valor generado para los propietarios de la empresa una vez descontado el coste del capital.

\subsection{Concepto de Valor Social Integrado dentro de la Contabilidad Social como nueva variable para realizar la Valoración de Empresas en las entidades no lucrativas}

El concepto a valorar se amplía, más allá de una visión estrictamente económico-financiera, y en especial en las entidades cuyo propósito está alejado de lo económico. Contribuir en esta línea es fundamental para demostrar qué valor generan y generarán aquellas entidades que no tienen por objeto la realización de actividad empresarial que requieren la consecución de transacciones económicas. En esta línea, la Contabilidad Social se ha mostrado útil para realizar valoraciones de empresas con perspectiva social, más allá de lo económico. En 
esta línea y revisando la realidad sobre la contabilidad social (véase Mathews, 1997), señalar que, si bien existen varios estudios que tratan de incluir en la década de los 70 la información para los stakeholders, se puede decir que principalmente Linowes (1972) es el que consolida el término que hace referencia a este tipo de información para los grupos de interés como Contabilidad Socio-Económica. Esto permite resolver cómo representar la imagen fiel de las entidades más allá de la contabilidad financiera convencional. En concreto las cooperativas francesas e italianas desarrollaron en la década de los 80 y 90 el balance cooperativo (RiahiBelkaoui, 1984; Vaccari, 1997), para que luego se extendiera a todo el conjunto de la sociedad. En una siguiente etapa, y a partir de todos estos trabajos, la contabilidad social evoluciona hacia la integración no solo de la información para los stakeholders, sino la inclusión de aspectos medioambientales o la aplicación de principios contables convencionales en los aspectos sociales (Gray, 2002).

En Retolaza y San-Jose (2018: 99) cuando se trata de establecer en qué organizaciones se debe aplicar señalan que "por su naturaleza, este tipo de generación de valor resulta evidente tanto en las entidades públicas como en las ONGs, pero también puede darse en las empresas mercantiles de todo tipo". Así, el desarrollo de la contabilidad social es incipiente, pero para las ENL es un aspectos diferencial y significativo ya que su principal propósito no reside en aspectos económicos sino sociales o altruistas (Retolaza et al., 2015), con lo que demostrar y explicar en qué consisten es fundamental por su razón de ser.

Esta contabilidad social se diferencia porque monetiza el valor social de las organizaciones a partir de la perspectiva de los stakeholders, es decir, de forma holística y con perspectiva del propio receptor de valor (véase Retolaza et al., 2016; San-Jose y Retolaza, 2016 y Retolaza y San-Jose, 2018, para una explicación completa). Consiste básicamente en, tras la realización de un mapa de stakeholders, realizar entrevistas personales para extraer a partir de la percepción de los stakeholders las variables de valor social que la organización genera. Posteriormente, estas variables se transforman en variables de valor social orientadas a indicadores para poder localizar los outputs y darles un valor a través de los proxys que serán quienes establezcan los valores monetarios unitarios. Todo ello se hará con objeto de conocer el valor social de no mercado, pero obviamente, entre el valor social de mercado tenemos valores claramente sociales. Por ello también una parte, a la cual denominamos valor social de mercado, se integrará con la de no mercado. Se eliminarán las duplicidades para lograr el valor social integrado (VSI) que nos permitirá conocer de forma anual el valor social generado por la organización. De esta forma se logrará dar a conocer la imagen fiel de la ENL, aplicando el principio de prudencia (menores valores garantizados y consolidados), el principio de fiabilidad (sólo se incorporará la información con fuentes de información fiables), el de trazabilidad (para conocer el proceso, los pasos, y el seguimiento de los valores de forma conjunta y de inicio a fin) o el principio de divulgación completa (se debe adjuntar toda la información desglosada que permita el correcto entendimiento del proceso y de la valoración final).

Existen en esta línea varios estudios publicados, entre ellos los relacionados con su aplicación en casos (Román et al., 2020; Retolaza et al., 2020; Lazkano y Beraza, 2019; Lazkano et al., 2019; Ayuso et al., 2020), los que tienen por objeto abordar la exposición sobre el análisis 
de las ratios a partir del valor social integrado (San-Jose et al., 2020), los que analizan su componente ético (Zicari, 2020) o aquellos que lo aplican a la compra pública (Bernal et al., 2019; Mendoza et al., 2019). Sin embargo, previamente no ha sido trabajado en el ámbito de la valoración de empresas, pero sin duda, y, sobre todo, para las ENLs tiene gran utilidad por poderse utilizar con objeto de los planes estratégicos o poder hacer un balance de su situación, entre otros. Así, en las próximas secciones resaltaremos su aplicabilidad y utilización.

\section{Estudio empírico 3.1. Método de Análisis}

El proceso se ha realizado utilizando un caso como ilustración para poder analizar la complejidad de aplicar métodos diferenciados de valoración de empresas en casos concretos y complejos (Forman y Damschroder, 2007), así es posible comprender situaciones de gran complejidad. Los estudios de caso se utilizan en investigación cuando los fenómenos sociales son complejos y cuando es necesario realizar un análisis profundo para comprender una realidad (Eisenhardt, 1989; Yin, 2013; Ruiz-Lozano et al., 2020). Se ha seleccionado el Museo de la Minería del País Vasco precisamente porque es un museo territorial, pequeño y con un propósito social muy claro. A continuación, exponemos en la Tabla 1 los datos más relevantes del Museo y del caso a utilizar con el propósito de lograr demostrar la utilidad para las ENL el uso del VSI como variable de valoración empresarial.

\subsection{Metodología para el cálculo del Valor Social Integrado (VSI)}

Siguiendo a Retolaza et al. (2016) y San-Jose y Retolaza (2016) se aplica la metodología SPOLY para lograr el Valor Social Integrado. Así, para llegar a la monetización del valor social, en primer lugar, se calculó el valor social generado por la actividad que la entidad desarrolla a través del mercado; en segundo lugar, el valor social propio generado en el marco del no mercado, entendiendo que la suma de ambas conforma el Valor Social Integrado anual. 


\section{Tabla 1. Ficha Técnica del Caso}

\begin{tabular}{|c|c|}
\hline $\begin{array}{l}\text { Características del análisis de } \\
\text { caso }\end{array}$ & Explicación \\
\hline Propósito de investigación & $\begin{array}{l}\text { Aplicación del VSI como variable de valoración de } \\
\text { empresas y en este caso de ENL }\end{array}$ \\
\hline Metodología de investigación & Estudio de caso de carácter holístico e ilustrativo \\
\hline Unidad de análisis & ENL cultural y patrimonial \\
\hline Ámbito geográfico & País Vasco \\
\hline Universo & ENL culturales y patrimoniales \\
\hline Tipo de muestra & $\begin{array}{l}\text { Muestra lógica que nos permite aplicar el modelo } \\
\text { analítico con objeto de comprobar la aplicación del vsI } \\
\text { en el contexto de la valoración empresarial. Selección } \\
\text { no aleatoria }\end{array}$ \\
\hline $\begin{array}{l}\text { Métodos de recogida de la } \\
\text { evidencia }\end{array}$ & $\begin{array}{l}\text { Revisión de Documentación: informes, web, artículos, } \\
\text { etc. } \\
\text { Realización de entrevistas a Stakeholders } \\
\text { Observación directa }\end{array}$ \\
\hline $\begin{array}{l}\text { Métodos de análisis de la } \\
\text { evidencia }\end{array}$ & Fundamentalmente de tipo cualitativo \\
\hline Enfoque científico & Proceso deductivo \\
\hline Fecha de realización & $\begin{array}{l}\text { Mayo-Septiembre } 2020 \text {, a partir de trabajos y } \\
\text { documentación previa }{ }^{2}\end{array}$ \\
\hline $\begin{array}{l}\text { CARACTERÍSTICAS DEL SUJETO DE } \\
\text { ANÁLISIS DE ESTUDIO. MUESTRA }\end{array}$ & Museo de la Minería del País Vasco \\
\hline Historia & 19 años \\
\hline Presupuesto & $\begin{array}{l}\text { Entre } 250.000 € \text { y } 275.000 \text { en los años de referencia, } \\
271.000 € \text { de media. }\end{array}$ \\
\hline Visitas por año & $\begin{array}{l}\text { Entre } 12.900 \text { y } 17.000 \text { visitas en los años de } \\
\text { referencia,15.000 visitas de media. }\end{array}$ \\
\hline Entrevistas a los Stakeholders & 12 \\
\hline Grabación & Sí \\
\hline Transcripción & Sí \\
\hline
\end{tabular}

Fuente: Elaboración propia.

2. Se han tomado como base los últimos datos disponibles en ese momento (ejercicio 2019), por lo que el efecto de la COVID-19 no ha sido tenido en cuenta. Este hecho no cuestiona la metodología seguida, aunque supondría cambios en el valor, que podrán estudiarse más adelante. 
El valor de mercado se calcula a partir de la contabilidad tradicional. Se detectan aquellas variables que tanto de forma directa como inducida generan valor social a la sociedad. Así, se incluirá la generación de valor generado por la contratación de trabajadores (salarios y seguridad social) y los impuestos (IVA, IRPF) desde la perspectiva directa. Para ello se ha utilizado principalmente el concepto desarrollado por la AECA (Asociación Española de Contabilidad y Administración de Empresas) para el Estado de Valor Añadido (EVA) y mediante la utilización de datos de la Cuenta de Pérdidas y Ganancias. Para la parte de valor generado inducido de mercado tras listar los proveedores se harán uso de estas variables en los proveedores, y se les asignarán como valor generado por la empresa que les contrata los porcentajes (la información es obtenida a partir de tener los CIFs de los proveedores y mediante el análisis de la información en la base de datos SABI mediante la cual se logran las partidas de sus cuentas anuales).

El valor de no mercado se logra tras la aplicación de cinco fases (SPOLY) (para una exposición detallada de las mismas consultar Retolaza et al., 2016). En la primera se fija el equipo y el cronograma, para en la segunda realizar la identificación de los grupos de interés a los que se les genera valor social, lo cual se plasma en un mapa de stakeholders. Posteriormente, en la fase tres, se realizan entrevistas para así poder conocer en qué consiste el valor social generado por la organización desde la perspectiva de cada uno de los grupos de interés. Una vez que constituimos el listado de variables de valor social y las agrupamos, en la cuarta fase, como se realiza en los ámbitos de calidad y en otras metodologías, nos centramos en cuantificar intersubjetivamente los outputs vinculados a cada variable de valor a través de proxys. Los proxys serán unidades monetarias asignadas a cada output o unidad de medida -hora, curso, actuación- y se aplicará siempre el principio de prudencia con objeto de no sobrevalorar ninguna actuación. La lógica utilizada en este proceso de cuantificación monetaria es la misma que la del valor razonable, con la diferencia que los rangos de valor en el caso de los intangibles son mucho más amplios y complejos de consensuar que en el caso de los bienes tangibles. Una vez cuantificados los outputs, en la última fase se procede al cálculo y visualización del valor social integrado, se agrupan el valor de no mercado y el de mercado para demostrar el VSI de la entidad.

\subsection{Metodología para el cálculo de la Valoración de entidades: el cálculo del Valor Social Esperado (VSE)}

De forma similar a como se calcula el valor económico de una empresa mercantil, queremos para las ENL realizar el cálculo de su valor, pero en este caso, la renta social será la clave de su actividad. Así, para calcular este valor, hemos utilizado el método de descuento de flujos de caja, que se utiliza de forma habitual en la valoración de empresas; con la peculiaridad de que en el cálculo hemos sustituido los flujos de caja económicos por los sociales, o lo que 
es lo mismo, por el valor social generado a lo largo de un periodo de tiempo (consistirá en la actualización del VSI).

Para ello ha sido necesario calcular las previsiones del Valor Social Integrado Neto para los próximos años, para lo que se han tomado como referencia los valores reales de los años 2017, 2018 y 2019.

Con esta información, se ha procedido a estimar el valor de estas mismas variables en los años 2020, 2021, 2022, 2023 y 2024, de cara a incorporar estos nuevos valores en la metodología de cálculo de Valor Social Integrado, y calcular las previsiones del mismo para 2020-2024.

Los supuestos utilizados para las proyecciones son los habituales en situaciones similares de valoración de empresas, y se detallan a continuación:

- En el caso de variables con una trayectoria reconocible (ventas e IVA asociado, gastos de personal, servicios exteriores): se ha estimado que el valor seguirá creciendo o decreciendo al mismo ritmo en los próximos años. El mismo proceso se ha utilizado para proyectar el Valor de No Mercado.

- En las variables sin tendencia clara (ayudas monetarias, aprovisionamientos, amortización de inmovilizado y porcentaje del mismo financiado por ayudas): se ha calculado el promedio en el período 2017-2019 y se ha mantenido como referencia. Este mismo criterio se ha aplicado en el caso de los índices de repercusión del gasto corriente a través de proveedores.

- Para las subvenciones imputadas al excedente del ejercicio: se ha estimado un crecimiento anual del 6\% (superior al 2,65\% observado), al tratarse de un aspecto clave tratado en la planificación, y necesario para el mantenimiento del Museo.

- Excepcionalmente, los datos no representativos en algún ejercicio, han sido excluidos, partiendo del valor y año que se considera extrapolable. Es el caso de los índices de repercusión para proveedores de inversión, para los que se ha utilizado el valor de 2017 (en 2018 no se dieron inversiones de este tipo, y en 2019 fueron extraordinarias). En cuanto al IVA soportado, se ha optado por tomar el valor de 2019, dado el cambio de criterio de este año, que se mantendrá en el futuro.

Más allá de las previsiones realizadas a 5 años, y siguiendo la práctica habitual en la valoración de empresas, se ha calculado un valor de continuidad o valor residual, partiendo de un mantenimiento del último valor social esperado (2024), y suponiendo que el mismo se mantendrá constante a partir de entonces. Normalmente se estima un valor constante o ligeramente creciente. En este caso, dado el bajo nivel de inflación, y tratando de evitar una sobrevaloración, se ha optado por un supuesto de renta constante con dos posibles escenarios: un período de 5 años (2025-2030) o 10 años (2025-2035), siendo conscientes de que posiblemente la generación de valor se alargue más en el tiempo (el Museo lleva ya más de 15 años funcionando).

Respecto a la tasa de actualización, ya se ha indicado la falta de acuerdo sobre la tasa idónea a aplicar y su variedad (Cruz Rimbaud y Muñoz Torrecillas, 2006), algo que también ocurre en las valoraciones de empresas al uso. En nuestro caso, se ha seguido el criterio fijado por 
Moore et al. (2004) para inversiones sociales con horizontes inferiores a 50 años en el marco de análisis Coste-beneficio, tomando un valor del 3,5\% anual.

Así, esta es la fórmula utilizada para el cálculo del Valor del Museo, a partir del Valor Social esperado para los próximos años.

$$
\text { Valor Museo }=\frac{V^{2020}}{(1+0,035)}+\frac{V^{2} I_{2021}}{(1+0,035)^{2}}+\cdots+\frac{V_{2024}+\text { Valor Renta Continuidad }}{(1+0.035)^{5}}
$$

El valor de la renta de continuidad se calcula como la actualización de flujos futuros constantes durante 5 ó 10 años (en función del supuesto). Para ello basta con aplicar la fórmula del valor de una renta unitaria

$$
a_{n \neg i}=\frac{1-(1+i)^{n}}{i}
$$

y multiplicarla por el valor de la renta de continuidad, que se considera constante. Dado que empieza a darse a partir de que finalicen las proyecciones, ese valor debe finalmente ser actualizado a 2020.

\section{Resultados}

\subsection{El caso de la Monetización de Valor del museo desde una perspectiva social}

El Museo de la Minería del País Vasco, reconocido como tal por la Ley de Museos de Euskadi, es una entidad sin ánimo de lucro, que adoptó la forma jurídica de fundación en el año 2001, y tal y como se establece en los estatutos, sus objetivos tienen una clara vocación de servicio a la sociedad en general ${ }^{3}$.

El Plan Director elaborado en el año 2011 señala que la razón de ser del Museo se basa en el estudio, la recuperación, preservación y difusión de la memoria histórica de la cultura minera del País Vasco dada su trascendencia histórica e identitaria. El marco de acción se sitúa principalmente en las cuencas mineras vizcaínas, de importantes y fructíferos cotos mineros, y que permitieron una continuidad de la actividad de la minería desde tiempos remotos, hasta finales del siglo XX (Museo de la Minería del País Vasco, 2011).

3. El estudio y la divulgación del conocimiento del desarrollo histórico de la actividad minera y de sus consecuencias económicas, sociales y medioambientales; la protección y recuperación de los elementos materiales, industriales, urbanos y de infraestructura procedentes de la actividad minera; la recuperación y conservación de documentos, archivos, planos, imágenes, dibujos y fotografías procedentes de la época y actividad minera, así como el estudio de los paisajes y elementos del medio natural en cuyo estado actual haya influido la actividad de la minería y la promoción del desarrollo socioeconómico de la zona minera de Vizcaya, teniendo en cuenta criterios de sostenibilidad. 
Entre sus aspiraciones se encuentra la de conseguir el reconocimiento institucional de la labor que realiza el Museo, con el objetivo de mejorar la relación con las instituciones públicas sin perder su identidad propia y autonomía. Esto le llevó a plantearse la necesidad de calcular y comunicar el valor de la entidad en términos monetarios de una manera singular, el reto residía en calcular un valor que fuera más allá de lo económico, capaz de reflejar de forma agregada la contribución económica y social que un proyecto cultural, como este, aporta a la sociedad en su conjunto.

Por ello, en el año 2016 el Museo de la Minería del País Vasco abordó un proceso formativo con la metodología action research denominado: "Action research Training: Valoración (Social) del Museo de la Minería del País Vasco", que tenía como objetivo incorporar el know-how de la metodología SPOLY para cuantificar el valor del Museo desde la perspectiva social. Los datos obtenidos en aquel momento han sido actualizados con el objetivo de presentar unos resultados adaptados al contexto actual. Así se han realizados los cálculos tomando como referencia los valores correspondientes a los últimos ejercicios cerrados, esto es 2017, 2018 y 2019.

\subsection{El Valor Social Integrado por el Museo de la Minería del País Vasco}

Se ha realizado el cálculo del VSI siguiendo la contabilidad social el valor de mercado a partir del estado de valor añadido propuesto por la AECA, y el valor de no mercado a partir de las cinco fases expuestas en la sección de metodología. A continuación, se exponen los principales resultados obtenidos de ese proceso.

\section{Valor de Mercado}

El valor de mercado (véase Tabla 2) del Museo de la Minería del País Vasco ha sido de $454.221,14$ € para el año 2017, 477.567,20 € para 2018 y 562.544,20 € para el año 2019. Se observa un crecimiento continuado para estos tres años analizados, aunque el aumento de 2019 (mayor) se da por una actividad no recurrente.

La lectura y explicación de estas variables calculadas es la siguiente:

- El valor socio-económico directo, generado directamente por la organización a través de aquellas operaciones que suponen movimientos de tesorería (transacciones económicas o pagos-cobros), es lo que llamaremos valor social de mercado directo (VES). También a partir de esta variable será posible realizar el cálculo de cuánto revierte a las administraciones públicas (R-VES). Así pues, el Museo de la Minería, a través de su actividad mercantil, generó en el año 2019, un valor para la sociedad de $248.328 €$, y un retorno para las administraciones públicas de $60.853 €$. 
Tabla 2. Valor de Mercado del Museo de la Minería del País Vasco

\begin{tabular}{l|rrc}
\multicolumn{1}{l|}{ Variables de la contabilidad social } & 2017 & 2018 & 2019 \\
\hline $\begin{array}{l}\text { Impacto directo de la actividad economica } \\
\text { Valor movilizado proveedores }\end{array}$ & $181.867,27 €$ & $205.344,54 €$ & $248.328,00 €$ \\
\hline $\begin{array}{l}\text { Valor movilizado proveedores de } \\
\text { inmovilizado }\end{array}$ & $24.895,49 €$ & $25.112,66 €$ & $17.828,21 €$ \\
\hline $\begin{array}{l}\text { Valor a clientes } \\
\text { Valor Social de Mercado [VES] }\end{array}$ & $10.346,37 €$ & $0,00 €$ & $46.721,99 €$ \\
\hline $\begin{array}{l}\text { VALOR ECONÓMICO SOCIAL Directo } \\
\text { Administración Pública (R-VES) }\end{array}$ & $237.112,00 €$ & $247.110,00 €$ & $249.666,00 €$ \\
\hline
\end{tabular}

- De forma similar se calculó el valor socio-económico indirecto de gasto corriente, generado por la actividad de sus proveedores, que ha sido inducido por los bienes entregados o los servicios prestados al museo, donde no se tiene en cuenta la totalidad del gasto, sino sólo el valor añadido en función de su distribución a la sociedad (salarios netos, retenciones de IRPF, Seguridad Social e Impuestos). En el año 2019 el valor social generado inducido a través de la actividad de sus proveedores de gasto corriente ascendió a $17.828,21 €$

- El valor socio-económico indirecto inducido por los proveedores de inmovilizado que proporcionaron bienes de inversión al museo en el año 2019 fue de 46.721,99 €. El valor inducido a través de los proveedores de inmovilizado no presenta un comportamiento uniforme y esto es debido a que las operaciones de inversión no son repetitivas y uniformes a lo largo de los años.

- Y finalmente, el generado en el entorno inducido por los usuarios de los servicios del museo en el año de referencia ascendió a 249.666,00 €.

\section{Valor de No Mercado}

Para identificar las variables que formarán parte del Valor social de no mercado, se formó un equipo de trabajo en el que participaban, por una parte, personas expertas del ámbito académico, y, por otra parte, personas voluntarias y salariadas con responsabilidad en la gestión del Museo. En una primera reunión se acordó el cronograma, las responsabilidades y los compromisos que adquirían cada una de las partes del equipo de trabajo. Posteriormente, se realizó un mapa de stakeholders, de los que se elegirían las personas que iban a ser entrevistadas. Finalmente, a partir de la información obtenida en las entrevistas, se determinaron las principales variables que generan valor social percibidas por los diferentes stakeholders. A 
continuación, se procedió a monetizar las variables, para lo cual se han utilizado los proxys. A continuación (véase Tabla 3), se presentan las variables que han sido identificadas como generadoras de valor por los distintos stakeholders, así como los proxys utilizados para monetizar el Valor social que se genera a partir de las actividades que no pasan por el mercado.

Tabla 3. Descripción de las Variables de Valor de No mercado del Museo

\begin{tabular}{|c|c|c|c|}
\hline Stakeholder & Variable de valor & Indicador & PROXY \\
\hline \multirow{9}{*}{ Usuarios } & Difusión cultura & № visitas & Precio entrada museo \\
\hline & $\begin{array}{l}\text { Memoria Histórica/ } \\
\text { Identidad }\end{array}$ & № consultas & $\begin{array}{l}\text { Coste persona } \\
\text { experta/hora }\end{array}$ \\
\hline & $\begin{array}{l}\text { Sostenibilidad } \\
\text { Medioambiental y } \\
\text { Social }\end{array}$ & № escolares & $\begin{array}{l}\text { Hora formación } \\
\text { en temáticas de } \\
\text { sensibilización } \\
\text { ambiental }\end{array}$ \\
\hline & Derechos Sociales & № visitas Web & $\begin{array}{l}\text { Valor de acceso a } \\
\text { páginas Web }\end{array}$ \\
\hline & $\begin{array}{l}\text { Cesión locales } \\
\text { para exposiciones } \\
\text { itinerantes }\end{array}$ & $\begin{array}{l}\text { № cesiones de } \\
\text { exposiciones } \\
\text { itinerantes }\end{array}$ & Informe profesional \\
\hline & Exposiciones & № exposiciones & Informe profesional \\
\hline & Charlas y conferencias & № charlas & Informe profesional \\
\hline & Cesión fotografías & $\begin{array}{l}\text { № cesión derechos } \\
\text { de imagen }\end{array}$ & Informe profesional \\
\hline & $\begin{array}{l}\text { Actividades } \\
\text { complementarias para } \\
\text { niños }\end{array}$ & № actividades & $\begin{array}{l}\text { Promedio del precio } \\
\text { de un parque infantil }\end{array}$ \\
\hline $\begin{array}{l}\text { Administraciones } \\
\text { públicas }\end{array}$ & $\begin{array}{l}\text { Tema común a la } \\
\text { mancomunidad: } \\
\text { Dinamización }\end{array}$ & № visitantes & $\begin{array}{l}\text { Cuestionario } \\
\text { específico }\end{array}$ \\
\hline $\begin{array}{l}\text { Administraciones } \\
\text { públicas }\end{array}$ & $\begin{array}{l}\text { Declaración biotopo } \\
(1.000 \mathrm{Ha})\end{array}$ & № hectáreas & $\begin{array}{l}\text { Informe } \\
\text { Arrendamientos }\end{array}$ \\
\hline $\begin{array}{l}\text { Administraciones } \\
\text { Públicas }\end{array}$ & Cesión Espacios & № de cesiones & Informe profesional \\
\hline $\begin{array}{l}\text { Administraciones } \\
\text { públicas }\end{array}$ & Imagen & $\begin{array}{l}\text { Aparición en } \\
\text { medios }\end{array}$ & $\begin{array}{l}\text { Valor } 1 / 2 \text { página en } \\
\text { prensa diaria }\end{array}$ \\
\hline \multirow{2}{*}{ Voluntariado } & $\begin{array}{l}\text { Recuperación } \\
\text { patrimonio cultural }\end{array}$ & $\begin{array}{l}\text { Horas } \\
\text { recuperación }\end{array}$ & $\begin{array}{l}\text { Informe de Empresa } \\
\text { Especializada }\end{array}$ \\
\hline & Ocio Activo & $\begin{array}{l}\text { Horas de } \\
\text { expediciones }\end{array}$ & $\begin{array}{l}\text { Informe de Empresa } \\
\text { Especializada }\end{array}$ \\
\hline $\begin{array}{l}\text { Entidades } \\
\text { Culturales }\end{array}$ & Cesión de materiales & $\begin{array}{l}\text { № cesiones de } \\
\text { materiales }\end{array}$ & $\begin{array}{l}\text { Informe de Empresa } \\
\text { Especializada }\end{array}$ \\
\hline
\end{tabular}




\section{Valor Social Integrado}

Una vez cuantificados los outputs se ha procedido a realizar el cálculo global, y se ha logrado visualizar el valor social integrado (VSI); mediante la suma del valor social de mercado y el de no mercado. La Tabla 4 resume los distintos componentes del valor social generado por el Museo de la Minería.

Tabla 4. Valor Social Integrado del Museo de la Minería del País Vasco

\begin{tabular}{l|rrr} 
& 2017 & 2018 & 2019 \\
\hline Valor Social de Mercado [VES] & $454.221,14 €$ & $477.567 .20 €$ & $562.544,20 €$ \\
\hline Valor Social No Mercado [VSNM] & $555.104,03 €$ & $566.474,03 €$ & $600.765,95 €$ \\
\hline Valor Social Integrado Bruto [VASIBruto] & $1.009 .325,17 €$ & $1.044 .041,23 €$ & $1.163 .310,15 €$ \\
\hline Financiación pública recibida & 269.854 & $247.110 €$ & $294.666,00 €$ \\
\hline Valor Social Integrado Neto [VASINeto] & $739.471,17 €$ & $796.931,23 €$ & $868.644,15 €$ \\
\hline
\end{tabular}

\subsection{Construcción del Valor del Museo desde una perspectiva social}

La obtención del Valor Social Integrado (renta) en un ejercicio concreto es interesante en sí mismo, y así lo demuestra el hecho de que cada vez son más las entidades lucrativas o sin ánimo de lucro que incluyen este tipo de información en sus Estados Contables o que tratan de calcularlo (como por ejemplo Lantegibatuak, CLADE, Mutualia o UCAN). Sin embargo, este proyecto tenía como objetivo determinar cuál sería el valor, expresado en términos monetarios, del Museo como entidad teniendo en cuenta las rentas sociales que es capaz de generar en un futuro. En el caso de entidades sin ánimo de lucro, que persiguen un interés general, deberían ser tenidas en cuenta no sólo las rentas económicas que es capaz de generar y que proporcionan movimientos en la tesorería de las entidades y finalmente se ven reflejadas en el PIB nacional, sino que deberían ser tenidos en cuenta, otros elementos intangibles y generadores de valor que la actividad del museo aporta a la sociedad, como son la generación de conocimiento, la sensibilización en temas sociales, ocupación del tiempo libre o reducción de impactos medioambientales, que, en última instancia, promueven una ciudadanía más culta y crítica que revierte en el bienestar de toda la sociedad.

En el caso del Museo de la Minería del País Vasco, considerábamos interesante obtener una valoración de la entidad en su conjunto, para comunicarlo y mejorar la percepción que desde las administraciones públicas se tiene de la entidad principalmente, pero también para demostrar a la sociedad qué se hace, y más importante aún cómo se hace. En el caso de las 
ENL, que dependen de las ayudas públicas para su pervivencia, es fundamental que exista una relación de confianza entre administración y entidad y un reconocimiento del valor que tienen las entidades a las que financian. Cada vez son más importantes para generar un bienestar social las alianzas público-privadas que tienen una vocación de permanencia. Por ello, resulta fundamental obtener una valoración de la entidad en su conjunto que sentará las bases para el establecimiento de convenios o partenariados con Administraciones locales o autonómicas, para el desarrollo y la promoción de actividades económicas, sociales y ambientales de interés general.

Con objeto de conocer el Valor del museo, el Valor Social Esperado (VSE) y aplicar las previsiones y actualizaciones expuestas en el apartado de metodología los resultados obtenidos son los siguientes (véase Tabla 5):

\section{Tabla 5. Valoración del Museo de la Minería desde una perspectiva social: VSE}

\begin{tabular}{lc}
\hline Valor Social Esperado (VSE) en la proyección a 5 años (actualizado a 2020) & 4.255 .448 \\
\hline Valor Social Esperado (VSE) en 10 años de continuidad (actualizado a 2020) & 7.198 .204 \\
\hline Valor Social Esperado Total con un horizonte de 15 años (actualizado a 2020) & 11.453 .652 \\
\hline
\end{tabular}

Con base en los datos de la tabla anterior, podemos concluir que el Valor del Museo sería de 11.453.652 €, a partir de los cálculos detallados de Valor Social esperado para los próximos 5 años, a los que se suma el valor de continuidad con un horizonte de otros 10 años. Debe tenerse en cuenta que el Museo lleva funcionando más de 15 años, y en todo momento se ha trabajado con supuestos prudentes, estimando siempre una continuidad del trabajo realizado en unos parámetros como los actuales.

\section{Conclusiones, limitaciones y futuras líneas de investigación}

La Valoración de Empresas es un ámbito de trabajo, tanto académico como profesional, con una larga trayectoria. Sin embargo, y a pesar del interés que pueda tener, su utilización en las entidades no lucrativas ha sido muy baja. Esto puede deberse al intento de analizar las rentas de las ENL del mismo modo en que lo hacen las entidades mercantiles, partiendo del valor económico-financiero, lo que hace muy limitada su utilización. Como veremos, en este trabajo se demuestra que es posible utilizar las rentas sociales como variables para conocer el valor 
de una entidad como tal, más allá de su generación de valor social en un período limitado (un año, por ejemplo).

Con esta finalidad, se ha utilizado el caso del Museo de la Minería del País Vasco, y se ha calculado el Valor Social Integrado de tres años (2017, 2018 y 2019) para luego proyectarlo a 5 ejercicios más, estimar un valor de continuidad, y realizar la necesaria actualización o descuento de flujos futuros. De esta forma la Valoración del Museo se ha elaborado a partir del Valor generado y esperado socialmente, y no el fundamentado únicamente en las variables económico-financieras. Esto permite utilizar las rentas sociales medidas mediante el VSI (valor de mercado y de no mercado) para establecer no solo qué está generando socialmente una entidad, sino qué puede generar a futuro. Su actualización nos da el Valor Social Esperado, el Valor del Museo como entidad, siendo esta una medida más acorde con el objeto del mismo, totalmente social. Así, concluimos no solo que es posible, sino que es útil la utilización de técnicas de valoración de empresas en ENL; y puede aprovecharse en términos de comunicación y gestión, así como para el refuerzo de la confianza hacia lo social, denotando compromiso con la sociedad.

Complementariamente, se propone una mayor amplitud de miras para estos procesos, y mediante el uso de la Teoría de Stakeholders y el VSI se ha incluido a todos los stakeholders como sujetos de interés de la valoración empresarial. Así, aportamos a las teorías de valoración de empresas su apertura hacia horizontes no exclusivamente económico-financieros sino también sociales. Por último, es preciso señalar que no debemos delimitar esta propuesta a las empresas sociales. En ese sentido, este trabajo abre una puerta a utilizar esta adaptación de la valoración de empresas no solo en ENLs, sino también en todo tipo de entidades, independientemente de su forma jurídica, su carácter social, su gobernanza o su adscripción pública.

Existen limitaciones para este trabajo, sobre todo porque solo se ha realizado en un caso, así como porque el VSI tiene limitaciones en sí mismo. Entre ellas se encuentra la que proviene del uso de los proxys, para monetizar las variables de valor social generado para los stakeholders. También porque quizá sea necesario analizar si existen otras variables de renta social que puedan bien aplicarse de forma complementaria, o bien sustituir a las propuestas. Además, cuestiones como la tasa de actualización a utilizar en la valoración de las ENLs pueden ser susceptibles de debate y, por ello, de un análisis en mayor profundidad.

En cuanto a las futuras líneas de investigación, parece necesario en primer lugar establecer mecanismos que consensuen los proxys a utilizar; para ello serán de gran utilidad los grupos de trabajos o comunidades de usuarios, en los que participen una muestra de las entidades de cada sector, para llegar a consensos sobre las cantidades a utilizar y aplicar. En segundo lugar, convendría lograr un mayor número de organizaciones que lo apliquen con objeto de confirmar que el Valor Social Esperado (VSE) es aplicable en organizaciones diferentes. Por último, señalar que se deberá analizar a futuro el cambio de perspectiva que supone para las ENLs realizar valoraciones de las entidades en términos sociales, ya que esto podría modificar el entendimiento global y ofrecer otros enfoques en cuanto a la generación de valor entre empresas mercantiles y de economía social, que hasta ahora solo estaban dirigidas por el valor económico. El VSE podrá así ser un mecanismo de comunicación, proyección o comparación global. 


\section{Referencias}

AYUSO, S., SÁNCHEZ, P., RETOLAZA, J.L. \& FIGUERAS-MAZ, M. (2020): “Social value analysis: the case of Pompeu Fabra University", Sustainability Accounting, Management and Policy Journal, 11(1), 233-252. DOI: 10.1108/SAMPJ-11-2018-0307.

BANCEL, F. \& MITTOO, U.R. (2014): "The gap between the theory and practice of corporate valuation: Survey of European experts", Journal of Applied Corporate Finance, 26(4), 106-117. D0I: $10.1111 /$ jacf.12095.

BERNAL, R., SAN-JOSE, L. \& RETOLAZA, J.L. (2019): "Improvement Actions for a More Social and Sustainable Public Procurement: A Delphi Analysis", Sustainability, 11(15), 4069: 9. D0I: $10.3390 /$ su11154069.

BOWMAN, W. (2009): "The economic value of volunteers to nonprofit organizations", Nonprofit Management and Leadership, 19(4), 491-506. D0I: 10.1002/nml.233.

CORDERY, C.J., PROCTOR-THOMSON, S. \& SMITH, K.A. (2011): "Valuing volunteer contributions to charities", Public Money and Management, 31(3), 193-200, D0I: 10.1080/09540962.2011.573230.

CRUZ RAMBAUD, S. \& MUÑOZ TORRECILLAS, M.J. (2006): "Social discount rate: a revision”, Anales de Estudios Económicos y Empresariales, 16, 75-98.

http://uvadoc.uva.es/handle/10324/19791.

DAMODARAN, A. (2016): Investment valuation: Tools and techniques for determining the value of any asset, John Wiley \& Sons, Hoboken.

EISENHARDT, K.M. (1989): "Building theories from case study research”, Academy of Management Review, 14(4), 532-550. D0I: 10.5465/amr.1989.4308385.

ETXEZARRETA, E., MENDIGUREN, J.C.P., DIAZ, L. \& ERRASTI, A. (2018): "Valor social de las cooperativas sociales: aplicación del modelo poliédrico en la cooperativa para la acogida de menores Zabalduz S. Coop", CIRIEC-España, Revista de Economía Pública, Social y Cooperativa, 93, 155-180. DOI: 10.7203/CIRIEC-E93.9953.

FERNÁNDEZ, P. (2019): "Métodos de valoración de empresas (Company Valuation Methods)", En: Valoración de empresas y sensatez, IESE Business School, Barcelona. D0I: 10.2139/ ssrn.1267987.

FORMAN, J. \& DAMSCHRODER, L. (2007): "Qualitative content analysis”, En: Jacoby, L. \& Siminoff, L.A. (Eds.), Empirical methods for bioethics: A primer, 39-62. JAI Press, Stamford. D0I: 10.1016/S1479-3709(07)11003-7. 
GÓMEZ-ZAPATA, J.D., ESPINAL-MONSALVE, N.E. \& HERRERO-PRIETO, L.C. (2018): “Economic valuation of museums as public club goods: Why build loyalty in cultural heritage consumption?", Journal of Cultural Heritage, 30, 190-198. DOI: 10.1016/j.culher.2017.09.010.

GRAY, R. (2002): "The social accounting project and Accounting Organizations and Society Privileging engagement, imaginings, new accountings and pragmatism over critique?", Accounting, Organizations and Society, 27(7), 687-708. DOI: 10.1016/S0361-3682(00)00003-9.

GUZMÁN, B., MENDOZA, J. \& PÉREZ, V. (2020): "El valor social y las cofradías canarias: una aproximación a través del análisis de un caso", CIRIEC-España, Revista de Economía Pública, Social y Cooperativa, 100, 115-153. DOI: 10.7203/CIRIEC-E.100.14486.

LAZKANO, L. \& BERAZA, A. (2019): "Social Accounting for Sustainability: A Study in the Social Economy", Sustainability, 11(24), 6894-20. D0I: 10.3390/su11246894.

LAZKANO, L. \& SAN-JOSE, L. (2020): “Monetización del valor social: el caso Clade”, Gizarte Ekonomiaren Euskal Aldizkaria = Revista Vasca de Economía Social, 16, 103-127. D0I: 10.1387/ reves.20903.

LAZKANO, L., SAN-JOSE, L. \& RETOLAZA J.L. (2019): "Social Accounting in the Social Economy: A Case Study of Monetizing Social Value”, En: FERRERIRA, A., MARQUES, R., AZEVEDO, G., INÁCIO, H. \& SANTOS, C. (Eds.), Modernization and Accountability in the Social Economy Sector, 132-151. IGI Global, Pensilvania. DOI: 10.4018/978-1-5225-8482-7

LINOWES, D.F. (1972): "Socio-economic accounting", Journal of Accountancy, 133, 37-42. https://www.jstor.org/stable/244751.

MATHEWS, M.R. (1997): "Twenty-five years of social and environmental accounting research: is there a silver jubilee to celebrate?", Accounting, Auditing and Accountability Journal, 10(4), 481-531. DOI: 10.1108/EUM0000000004417.

MENDOZA, J., HERNANDEZ, M. \& FRANCO, S.E. (2019): "Sustainable Public Procurement: From Law to Practice", Sustainability, 11(22), 6388. D0I: 10.3390/su11226388.

MOORE, M.A., BOARDMAN, A.E., VINING, A.R., WEIMER, D.L. \& GREENBERG, D.H. (2004): “'Just give me a number!' Practical values for the social discount rate", Journal of Policy Analysis and Management, 23(4), 789-812. DOI: 10.1002/pam.20047.

MUSEO DE LA MINERÍA DEL PAÍS VASCO (2011): Plan Director del Museo de la Minería del País Vasco. Recuperado de http://www.meatzaldea.eus/images/cuentas/PLAN\%20DIRECTOR. pdf.

RETOLAZA, J.L., AGUADO, R. \& SAN-JOSE, L. (2020): "Social Accounting as an Enabling Tool to Develop Collective Organizational Citizenship Behavior in the Diocese of Bilbao", Frontiers in Psychology, 11(77), 1-11. D0I: 10.3389/fpsyg.2020.00077. 
RETOLAZA, J.L. \& SAN-JOSE, L. (2018): “Contabilidad Social para el Bien Común”, Revista de Responsabilidad Social de la Empresa, 29(2), 95-122.

RETOLAZA, J.L., SAN-JOSE, L. \& RUIZ-ROQUEÑI, M. (2015): “Monetarizing the social value: theory and evidence", CIRIEC-España, Revista de Economía Pública, Social y Cooperativa, 83, 43-62.

RETOLAZA, J.L., SAN-JOSE, L. \& RUÍZ-ROQUEÑI, M. (2016): Social accounting for sustainability Monetizing the social value, Springer, Cham. DOI: 10.1007/978-3-319-13377-5.

RIAHI-BELKAOUI, A. (1984): Socio-economic accounting, Praeger Pub Text.

ROMÁN, C., GUZMÁN, B., MENDOZA, J. \& PÉREZ, M.V. (2020): "La sostenibilidad social de las SATs: una propuesta de indicadores para su evaluación", REVESCO, Revista de Estudios Cooperativos, 133, 71-80. D0I: 10.5209/reve.67336.

RUIZ-LOZANO, M., TIRADO-VALENCIA, P., SIANES, A., ARIZA-MONTES, A., FERNÁNDEZ-RODRÍGUEZ, V. \& LÓPEZ-MARTÍN, M.C. (2020): "SROI Methodology for Public Administration Decisions about Financing with Social Criteria. A Case Study", Sustainability, 12(3), 1070. DOI: 10.3390/su12031070.

SAN-JOSE, L. \& RETOLAZA, J.L. (2016): Contabilidad Social orientada a los stakeholders. Perspectiva de la Administración Pública, Ediciones Pirámide, Madrid.

SAN-JOSE, L., RETOLAZA, J.L. \& BERNAL, R. (2020): “Índice de valor social añadido: una propuesta para analizar la eficiencia hospitalaria”, Gaceta Sanitaria, 35(1), 21-27. D0I: 10.1016/j. gaceta.2019.08.011.

TESSLER, A., GAMBARIN, A. \& LUKINS, S. (2019): Value study of galleries, libraries, archives and museums (GLAMs) in Canada, Oxford Economics, Oxford.

VACCARI, A. (1997): “Constructing the Social Balance: Consumer Cooperative Italy", En: Zadek, S., Pruzan, P. \& Evans, R. (Eds.), Building Corporate Accountability, Earthscan, London.

WNUCZAK, P. (2018): "Social value added (SVA) as an adaptation of economic value added (EVA) to the specificity of cultural institutions", Journal of Management and Business Administration. Central Europe, 26(1), 100-120. DOI: 10.7206/jmba.ce.2450-7814.221.

YIN, R.K. (2013): "Validity and generalization in future case study evaluations", Evaluation, 19(3), 321-332. DOI: 10.1177/1356389013497081.

ZICARI, A. (2020): "The many merits and some limits of Social Accounting: why disclosure is not enough", En: San-Jose, L., Retolaza, J.L. \& Van Liedekerke, L. (Eds.), Handbook on Ethics in Finance, Springer, Cham. DOI: 10.1007/978-3-030-00001-1_14-1. 